DE DE GRUYTER

OPEN
Journal of Intercultural Management

Vol. 5, No. 2, June 2013, pp. 73-89

DOI 10.2478/joim-2013-0011

José Luis Benítez Contreras

Universidad de Guadalajara

\title{
La Inmersión en la Economía del Conocimiento de las Ciudades Medias del Centro-Occidente de México 2000-2010
}

\begin{abstract}
The knowledge-based economy is a new economy growth theory that for some authors is the new alternative for the economy growth. In this sense arise the medium cities as an alternative to economic growth, as these cities do not have the problems of the big cities and it is assumed that in the long term these medium cities will become large cities.

Thus, the objective of this research was to find that medium cities of west-central Mexico are embedded within the knowledge economy through the comparative analysis between the Census of Population and Housing 2000 and 2010 conducted by the Institute national Statistics and Geography (Instituto Nacional de Estadística y Geografía, INEGI). The region of west-central Mexico by the end of this research is comprised by the states of Aguascalientes, Colima, Guanajuato, Jalisco, Michoacán, Nayarit, San Luis Potosi and Zacatecas. Of those states were found that there are 39 medium cities. The result is not satisfactory as was found that there are 13 cities not embedded within the knowledgebased economy.
\end{abstract}

Key words: Economy, knowledge, medium cities

Resumen: La economía del conocimiento es una nueva teoría del crecimiento económico que para algunos autores es la nueva alternativa para el crecimiento económico. En este sentido surgen las ciudades medias como una alternativa para el crecimiento económico, puesto que estas ciudades no poseen los problemas de las ciudades grandes y se asume que en el largo plazo las ciudades medias crecerán hasta convertirse en ciudades grandes.

Es así que el objetivo de esta investigación fue comprobar que las ciudades medias del centro-occidente de México se encuentran inmersas dentro de la economía del 
conocimiento a través del análisis comparativo entre los datos del Censo de Población y Vivienda 2000 y 2010 que realizó el Instituto Nacional de Estadística y Geografía (INEGI).

La región del centro-occidente de México para fines de esta investigación está comprendida por las entidades federativas de Aguascalientes, Colima, Guanajuato, Jalisco, Michoacán de Ocampo, Nayarit, San Luis Potosí y Zacatecas. De dichas entidades federativas se encontró que hay 39 ciudades medias. El resultado obtenido no es satisfactorio dado que existen 13 ciudades medias que se determinó que no se encuentran dentro de la economía del conocimiento.

Palabras clave: Economía, conocimiento, ciudad media

\section{Introducción}

Para algunos autores la economía del conocimiento viene a reemplazar el estilo de producción e innovación de la economía, donde el capital humano será el principal activo, por lo que los conocimientos que posea dicho capital serán altamente importantes para el crecimiento económico de un país o de una región. [Ahrweiler, Pyka \& Gilbert, 2011; Chivu, Popescu, Scarlat, CiocarlanChitucea \& Popescu, 2010; Kriđčiűnas \& Daugëlienë, 2006; Melkinas, 2009; Rybakovas, 2010; Sîrbu, Doinea \& Mangra, 2009; Toma, 2010; Welfe, 2008; Zientara, 2008].

Si bien, algunas teorías indican que las ciudades grandes serán quienes tendrán mayor éxito dentro de la economía del conocimiento dado que poseen mejor infraestructura, mayor población, más instituciones de educación, más capital humano y mayor concentración de empresas; existen algunos estudios que demuestran que no es regla [Jordan \& Simioni, 1998]. Uno de dichos estudios es el realizado en Canadá [O’Hagan \& Rutland, 2008].

Es así que es importante analizar la situación de las ciudades medias de México y comprobar si en efecto, la economía del conocimiento sólo se desarrolla de manera favorable en las ciudades grandes, o como se propone en esta investigación, también las ciudades medias están generando un crecimiento económico a través del desarrollo de la economía del conocimiento.

Por lo tanto es indispensable analizar la situación de México en la inserción hacia la economía del conocimiento, partiendo de que las ciudades medias eventualmente evolucionaran hasta convertirse en las próximas grandes ciudades o metrópolis de México. Por lo que para motivos de esta investigación se propone un estudio de la situación de las ciudades medias del centro-occidente de México con respecto a la economía del conocimiento a través de un análisis comparativo entre el escenario dado por el Censo de Población y Vivienda 2010 y el Censo de Población y Vivienda 2000 para conocer la evolución y su desarrollo dentro de la economía del conocimiento. 


\section{La economía del conocimiento, definición y características}

La Organización para el Cooperación y el Desarrollo Económico [1996, p. 9] define a la economía del conocimiento como: "la economía basada en la producción, distribución y uso del conocimiento y de la información."

Otra de las primeras definiciones para la economía del conocimiento es la que Castells [1999, p. 2] proporciona, quien dice que la economía del conocimiento:

Es un fenómeno nuevo porque sólo en las dos últimas décadas del siglo XX se ha constituido un sistema tecnológico de sistemas de información, telecomunicaciones y transporte, que ha articulado todo el planeta en una red de flujos en las que confluyen las funciones y unidades estratégicamente dominantes de todos los ámbitos de la actividad humana.

Por su lado, Peluffo \& Catalán [2002, p. 10] proponen la definición de economía basada en el conocimiento y el aprendizaje de la siguiente manera: "es un sistema en el cual el motor de creación de valor y/o beneficios es el conocimiento y la capacidad para construirlo por medio del aprendizaje". Cabe resaltar que dichos autores manejan el aprendizaje como una variable aparte del conocimiento, sin embargo para motivos de esta investigación, la variable del aprendizaje ira inmersa en el conocimiento, ya que se considerará como parte integral del conocimiento.

Medina \& Ortegón [2006, p. 21] proponen la misma definición de economía del conocimiento propuesta por Kuznetsov \& Dahlman et al. [2008], quienes lo define como: "aquella economía que estimula a sus organización y personas a adquirir, crear, diseminar y utilizar el conocimiento de modo más efectivo para un mayor desarrollo económico y social". Así mismo, Kriđčiúnas et al. [2006] proponen como definición de economía del conocimiento la propuesta por Kuznetsov et al. [2008].

Rybakovas [2010, p. 68] propone la siguiente definición para economía del conocimiento: "es aquella economía que utiliza el conocimiento como el motor principal para su crecimiento económico. Es una economía donde el conocimiento es adquirido, creado, diseminado y utilizado de forma efectiva para mejorar su desarrollo económico".

Barković, I., Borozan, Đ., \& Dabić, M. [2008, p. 30] conceptualizan a la economía del conocimiento como:

Aquella donde la generación y la explotación del conocimiento juegan una parte predominante en la creación de la riqueza. No es una economía de escasez, sino de abundancia porque la información y el conocimiento pueden ser compartidos y actualmente crecen a través de su aplicación. Un componente clave en la economía del conocimiento integrado en las personas es el capital humano y la tecnología. 
Ahora bien, las características de la economía del conocimiento son diversas. Si bien se ha considerado como un nuevo sistema económico de crecimiento [Ferraro, 1999], su nacimiento es a partir de la era pos-industrial, es decir, alrededor de la década de 1980. Por ello, Medina et al. [2006, p. 23] indican que las principales características de la economía del conocimiento serían tres:

1. El conocimiento es el factor clave del desarrollo en la economía global. Hace la diferencia entre riqueza y pobreza.

2. La creación y redistribución de la riqueza básica para que compita exitosamente una sociedad implica la necesidad de promover la redistribución del conocimiento.

3. La multiplicación o explosión del conocimiento aumenta la necesidad de educación masiva y pertinente de la población.

Soria \& Aboites [2008] afirman que las diferencias en los tipos de conocimientos provienen de su misma naturaleza, dado que el conocimiento es un bien económico parcialmente no-excluyente y no-rival en el consumo. Por su parte, Entonces, Sîrbu et al. [2009, p. 229] argumentan que:

La economía del conocimiento se caracteriza principalmente por la transformación de conocimiento en materia prima, capital, productos y en factores esenciales para la producción de la economía; por los procesos económicos a través de los cuales la generación, compra, venta, estudio, depósito, desarrollo, distribución y protección de los conocimientos se convirtió en lo predominante e influye decisivamente en el logro de ganancias y el seguro de sostenibilidad de la economía en el largo plazo.

En resumen, la característica principal de la economía del conocimiento, es en sí, el conocimiento mismo, siempre y cuando éste sea el factor productivo predominante como factor de crecimiento económico ,o bien, que exista un valor-conocimiento ${ }^{1}$ dentro del producto final [Sakaiya, 1995]. Si bien hay otras características que separan a la economía del conocimiento de las demás teorías de crecimiento económico, la más importante es que el conocimiento sea el motor para el desarrollo sustentable de una economía.

\section{Índices de organismos mundiales de la economía del conocimiento}

Medina et al. [2006, p. 64] comentan que: "estos índices o e-readiness buscan cuantificar la preparación o nivel de avance de un país para la sociedad de la

1 Sakaiya (1995) explica el valor-conocimiento como una creación de los seres humanos, dado que es un aspecto singular de la sociedad del conocimiento que la distingue de la sociedad industrial, en la cual la tendencia es unir la mano de obra con los medios de producción. En pocas palabras, el valor-conocimiento es el valor agregado que lleva cualquier producto simplemente por el factor conocimiento que lleva en su elaboración, es decir, mientras que los componentes para producir cierta mercancía pueden ser baratos, el factor conocimiento eleva su precio final. Por ejemplo: la patente para elaborar cierto medicamento, mientras los componentes pueden ser baratos, la fórmula y el proceso de elaboración puede otorgarle un valor agregado que tiene como consecuencia el aumento en el precio final del medicamento hacia el consumidor final. 
información y del conocimiento". Para lo que hacen mención de los índices más famosos que existen, los cuales se muestran junto con una pequeńa descripción de cada uno en la Tabla 1

Así mismo, Medina et al. [2006, p. 65] afirman que: "todos los anteriores índices en cierta medida dan cuenta de los progresos y ubicación de cada país respecto a la ciencia, a la tecnología, la innovación y la transición hacia una Sociedad del Conocimiento".

Tabla 1. Índices más famosos de la economía del conocimiento.

\begin{tabular}{|c|c|}
\hline Nombre del índice & Descripción \\
\hline Digital Access Index (DAI) & $\begin{array}{l}\text { Mide el acceso a las tecnologías de la información y la } \\
\text { comunicación (TIC), incluye ocho indicadores agrupados en } \\
\text { cinco categorías y cubre } 178 \text { países. }\end{array}$ \\
\hline $\begin{array}{l}\text { Digital Opportunity Index } \\
\text { (DOI) }\end{array}$ & $\begin{array}{l}\text { Mide las oportunidades digitales de la sociedad de la } \\
\text { información y uso de las tecnologías (TIC). Utiliza } 11 \\
\text { indicadores agrupados en tres categorías (oportunidad, } \\
\text { infraestructura y uso). }\end{array}$ \\
\hline $\begin{array}{l}\text { Index of Knowledge } \\
\text { Societies (IKS) }\end{array}$ & $\begin{array}{l}\text { Mide la evolución y el comportamiento de los países en el logro } \\
\text { de una sociedad del conocimiento. Trabaja con } 14 \text { indicadores } \\
\text { cuantitativos organizados en } 3 \text { categorías (activos, avances y } \\
\text { prospectiva) y abarca } 45 \text { países. }\end{array}$ \\
\hline $\begin{array}{l}\text { Knowledge Economic Index } \\
\text { (KEI) }\end{array}$ & $\begin{array}{l}\text { Trata de mostrar las fortalezas y debilidades de los países en } \\
\text { la transición hacia la sociedad del conocimiento. Emplea } 12 \\
\text { indicadores agrupados en cuatro categorías. }\end{array}$ \\
\hline $\begin{array}{l}\text { Network Readiness Index } \\
\text { (NRI) }\end{array}$ & $\begin{array}{l}\text { Mide la preparación de un país para participar de los } \\
\text { beneficios de los avances de las tecnologías de la información } \\
\text { y el conocimiento. Incluye a } 104 \text { países con } 51 \text { indicadores } \\
\text { cuantitativos y cualitativos, divididos en } 3 \text { categorías. }\end{array}$ \\
\hline $\begin{array}{l}\text { Technological Achievement } \\
\text { Index (TAI) }\end{array}$ & $\begin{array}{l}\text { Los informes del PNUD sobre Desarrollo Humano incluye este } \\
\text { Índice como reflejo de la capacidad tecnológica de un país. }\end{array}$ \\
\hline UNCTAD Index (ICT) & $\begin{array}{l}\text { Esta diseńado para medir y comparar el desarrollo de las } \\
\text { tecnologías de la información y el conocimiento (conectividad, } \\
\text { acceso y políticas). Incluye indicadores cualitativos y } \\
\text { cuantitativos agrupados en estas tres categorías. }\end{array}$ \\
\hline UNPAN Index (UNPAN) & $\begin{array}{l}\text { A cargo de la Division for Public Administration and } \\
\text { Development Management de las Naciones Unidas, mide } \\
\text { el comportamiento de los países de acuerdo al desarrollo } \\
\text { de los Sitios Web, infraestructura de telecomunicaciones y } \\
\text { capacidades humanas. El ranking se realiza sobre } 178 \text { países y } \\
\text { también se le conoce como e-government index. }\end{array}$ \\
\hline $\begin{array}{l}\text { Índice del Banco Mundial } \\
\text { (WSIS) }\end{array}$ & $\begin{array}{l}\text { Este Índice incluye } 15 \text { indicadores distribuidos en } 5 \text { categorías } \\
\text { (acceso, calidad, sostenibilidad, aplicaciones y provisión) de las } \\
\text { TIC en } 144 \text { países. }\end{array}$ \\
\hline
\end{tabular}




\begin{tabular}{|l|l|}
\hline ArCo & $\begin{array}{l}\text { En honor a sus autores Daniel Archibugi y Alberto Coco, es una } \\
\text { derivación del Índice de Logros Tecnológicos (TAI) del PNUD e } \\
\text { incluye indicadores de creación tecnológica, infraestructura y } \\
\text { habilidades de recursos humanos. }\end{array}$ \\
\hline
\end{tabular}

Fuente: Elaboración propia con datos de Medina et al. [2006, p. 65].

Por su parte, la OCDE [1996, p. 30] indica que:

Hay cuatro razones principales por las que los indicadores de conocimiento, sin embargo, aunque sean cuidadosamente construidos, no puede aproximarse a la amplitud sistemática de los indicadores económicos tradicionales:

1. no hay fórmulas estables o "recetas" para la traducción de los insumos en la creación de conocimiento en productos de conocimiento;

2. la creación de conocimiento es difícil de trazar porque no hay cuentas análogas de conocimiento para las cuentas nacionales tradicionales;

3. el conocimiento carece de un sistema de precios sistemático que sirva como base para agregar piezas de conocimiento que son esencialmente únicas;

4. la creación de nuevo conocimiento no es necesariamente una adición neta al stock de conocimiento, y la obsolescencia de las unidades del stock de conocimientos no está documentado.

Así mismo, la OCDE [1996, p. 31] indica que sus principales indicadores de conocimiento, recolectados y estandarizados por ella son: “i) los gastos en investigación y desarrollo (I+D), ii) el empleo de ingenieros y personal técnico, iii) las patentes, y iv) balanzas de pagos de la tecnología”.

\section{Las ciudades medias, definición y características}

\section{Cuervo [2006, p. 23] define a la ciudad como:}

Un microcosmos del espacio social y condensa sus características; no obstante, posee rasgos que la hacen particular, que la especifican: la densidad de los elementos y la intensidad de las interacciones generan diferencias cuantitativas y cualitativas en el comportamiento de la ciudad como componente particular, pero dominante, del espacio social.

Zoido; De la Vega; Morales; Mas \& Lois [2000] definen a la ciudad como:

"Ciutat / Hiri / Cidade. Núcleo de población de ciertas dimensiones y funciones especializadas en un territorio amplio."

Sabatini [1998, p. 148] indica que:

Definir conceptualmente ciudad intermedia es difícil, casi imposible. En buena medida ello es así porque es difícil definir ciudad. Todos concordamos que una ciudad es un asentamiento con un tamańo, una densidad y una heterogeneidad social y funcional mínimas. El problema es determinar en términos prácticos qué es lo mínimo. Y cuando intentamos definir ciudad por su función respecto del espacio que no queda 
comprendido en ella -como se intenta hacer con la ciudad intermedia- entonces la dificultad aumenta. La ciudad como hecho físico no coincide, no es "simétrica" con la ciudad como hecho social (cultural, económico, político).

Por sulado, Macció [1985, p. 52] indica que: "todo conjunto de casas habitables agrupadas se llama aglomeración de población, cualquiera sea su tamańo”. Por lo tanto, define a la ciudad como: "una aglomeración en cuya vida económica no predomina la actividad agrícola”. [Macció, 1985, p. 52]

Así mismo, Macció [1985, p. 52] menciona que: "las aglomeraciones urbanas se forman generalmente por integración de localidades o municipios limítrofes, que en algunos casos siguen conservando cierto grado de autonomía administrativa."

Cabrales \& Canosa [2001, p. 4] definen a la ciudad como: "espacios de integración y de promoción social, aunque en su mayor parte estén compuestas de zonas desfavorecidas, mal equipadas y en situación irregular".

Jordán et al. [1998, p. 225] indica que: "se puede asumir que un asentamiento intermedio es todo aquel que por no constituir área metropolitana o pequeńo núcleo rural, ofrece un equilibrio entre las oportunidades que genera la ciudad y las desventajas o deseconomías de un asentamiento metropolitano no controlado."

Por lo tanto, para fines de esta investigación, se tomará la definición de ciudad de Cuervo [2006, p. 23]. Así mismo, se complementa con la definición de Jordán et al. [1998, p. 225] respecto a la definición de ciudad media.

Ahora bien, algunas características de las ciudades medias de acuerdo a Cuervo [2003, p. 44] es que proporciona una razón para denotar una de las ventajas de las ciudades medias, siendo las características de las grandes ciudades latinoamericanas un área de oportunidad para las ciudades medias. Así como una característica de las ciudades medias, siendo éstas de menor tamańo su característica puede denotarse como su conexión con los sectores locales.

Schneier [1990, p. 347] menciona una característica de la ciudad por lo que dice que:

Desde su inicio y especialmente en el siglo XX, las ciudades han estado en la arena del experimento social y el desarrollo de nuevas ideas politicas y culturales. La comprensión de la situación de las ciudades, no obstante, presupone un mayor entendimiento de la crisis que afecta al Estado-nación, el modelo de acumulación de capital y las relaciones entre el Estado y la sociedad que han prevalecido durante los últimos 30-40 ańos, especialmente en las ciudades.

Ahora bien, el Banco Mundial [2000, p. 1] indica como característica de las ciudades que: "en los umbrales del siglo XXI, las ciudades y los pueblos constituyen la línea de avanzada de la campańa en pro del desarrollo. [...] Las 
ciudades y los pueblos no sólo crecen en tamańo y número; también están adquiriendo mayor influencia".

Otra característica de todas las ciudades es que el gobierno local, ya sea de un Estado Federal o de un Estado unitario, continua siendo el rostro cotidiano del sector público hacia la sociedad.[Banco Mundial, 2000, p. 2].

Así mismo, Cabrales et al. [2001, p. 4] dan algunas características de las ciudades medias, las cuales son:

Su sorprendente capacidad para integrar a los pobres a través de formas de clientelismo y un poderoso imaginario político ha caracterizado a estas ciudades durante los ańos de crecimiento, que van de la década de los ańos cuarenta a la de los sesenta del siglo $X X$. Desde los ańos ochenta, esta imagen integradora de la ciudad latinoamericana parece no tener ya sentido aunque exista una tendencia a la democratización. El agotamiento de ese esquema estaría determinado por el aumento de la pobreza y la violencia que originan nuevas formas de segregación desde el momento en que se buscan mecanismos efectivos que garanticen la seguridad.

Por último, Balbo [1998, p. 118] menciona la siguiente característica de las ciudades de América Latina: "En América Latina, como en la mayoría de las demás regiones, las ciudades se han vuelto en el centro y el motor del desarrollo".

\section{Las ciudades del conocimiento: El desarrollo local a través de la economía del conocimiento}

Carrillo Gamboa [2011] define a las ciudades del conocimiento como: „comunidades urbanas donde la ciudadanía emprende un esfuerzo deliberado y sistemático por desarrollar su sistema de capitales en forma balanceada y sostenible."

Romeiro \& Méndez Gutiérrez Del Valle [2008] indican que: "a pesar de que el conocimiento siempre ha sido considerado un importante factor de progreso, esta denominación -ciudad del conocimiento- es relativamente reciente, aludiendo a una intencionalidad en su gestión como factor estratégico para el desarrollo".

Por ello, dichos autores definen a la ciudad del conocimiento como: "aquella que desarrolla estrategias intencionales con el objetivo de intensificar la producción, transferencia y aplicación del conocimiento y procura incentivar una cultura de aprendizaje colectivo y de difusión del conocimiento entre todos los actores (ciudadanos, organismos públicos, empresas, etc.)".

Por lo tanto cabe mencionar que un rasgo distintivo de una ciudad del conocimiento sería la prioridad que concede a la inversión asignada a la educación, la formación y la investigación [Romeiro et al., 2008].

Ding \& Zhao [2011, p. 48] ayudan a entender mejor la relación entre el crecimiento económico y el desarrollo local dado que partiendo desde su óptica, 
es como se puede explicar la creación de cluster's para generar empleo, dado que son beneficiosos para la creación, transferencia y uso del conocimiento, por lo que dicen que:

La relación entre forma urbana y el crecimiento económico urbano está mediada por las economías de aglomeración que facilitan encontrar mano de obra, reducir los costos de producción en la industria manufacturera y el transporte, y promover los derrames en la tecnología y la administración.

Por lo tanto, la presencia de elementos positivos o impulsores en una región no significa que exista una relación con el resto de los elementos que genere competitividad. Por ejemplo, la presencia de universidades y centros de investigación no necesariamente crea vínculos estables con la industria [Celis, 2009, p. 183].

Entonces se puede concluir que el desarrollo local y la economía del conocimiento, si bien, son fenómenos que se estudian de manera separada, también ofrecen la posibilidad de estudiarlos de forma conjunta. El desarrollo local demuestra ser un conjunto de varios factores, mientras que la economía del conocimiento es meramente una herramienta para el desarrollo local, dado que sólo es una teoría de crecimiento económico. Por ello es posible afirmar que el desarrollo local se puede dar a través de la economía del conocimiento, aunque existen desafíos también hay áreas de oportunidad para lograrlo, lo cual se abordará en el siguiente apartado.

\section{Resultados}

Las entidades federativas que se consideraron para la elaboración de este estudio son: Aguascalientes, Colima, Guanajuato, Jalisco, Michoacán de Ocampo, Nayarit, San Luis Potosí y Zacatecas; dando un total de 8 entidades federativas. Ahora, el conjunto de las 8 entidades federativas dan un total de 441 municipios y un total de $24,184,807$ habitantes. $^{2}$

Ahora bien, para elegir las ciudades medias del centro-occidente de México se aplicó la Ley Zipf. Lo cual consiste en aplicar LOG a la población de cada municipio para con ello conocer el valor de "Y" y ordenar los municipios del mayor al menor en cuanto a su población, para obtener el Rango, teniendo el mayor un rango con valor a cero y con ello obtener el valor de "X" [Cuervo, 2004]. Con los datos recopilados se obtiene la gráfica 1.

Cabe mencionar que la interpretación de la Gráfica 1 se ve limitada por el espacio, no obstante, con base en los valores que tiene cada municipio, de acuerdo a este método, las ciudades medias serán las que tiene un LOG Municipio igual 5. Si bien se pudo haber considerado como ciudades medias a todos aquellos

2 Datos obtenidos a través de los Anuarios Estadísticos edición 2011 de cada entidad federativa. También se puede consultar el portal de internet de INEGI, donde de igual manera se encuentran los datos disponibles. 
municipios que tuvieron un LOG Municipio igual a 5 y a 4 , dado que son las que quedan en la escala media, también es importante tomar en consideración que si se examina la población existente en los municipios con LOG Municipio igual a 4 se podrá observar que la densidad demográfica es menor a cien mil habitantes, para lo que INEGI [2012a] indica que:

Para la variable Tamańo Localidad los rubros corresponden a:

- Áreas más urbanizadas corresponde a Localidades de 100,000 y más habitantes.

- Áreas menos urbanizadas corresponde a Localidades menores de 100,000 habitantes.

- Urbano medio corresponde a Localidades de 15,000 a 99,999 habitantes.

- Urbano bajo corresponde a Localidades de 2,500 a 14,999 habitantes.

- Rural corresponde a Localidades menores de 2,500 habitantes.

Por lo tanto se consideran a los municipios con más de 100,000 habitantes, es decir, las áreas más urbanizadas, puesto que esto se considera desde el supuesto que una ciudad debe ser parte de la jerarquía de Áreas más urbanizadas.

Gráfica 1. Ley Zipf aplicada a los 441 municipios

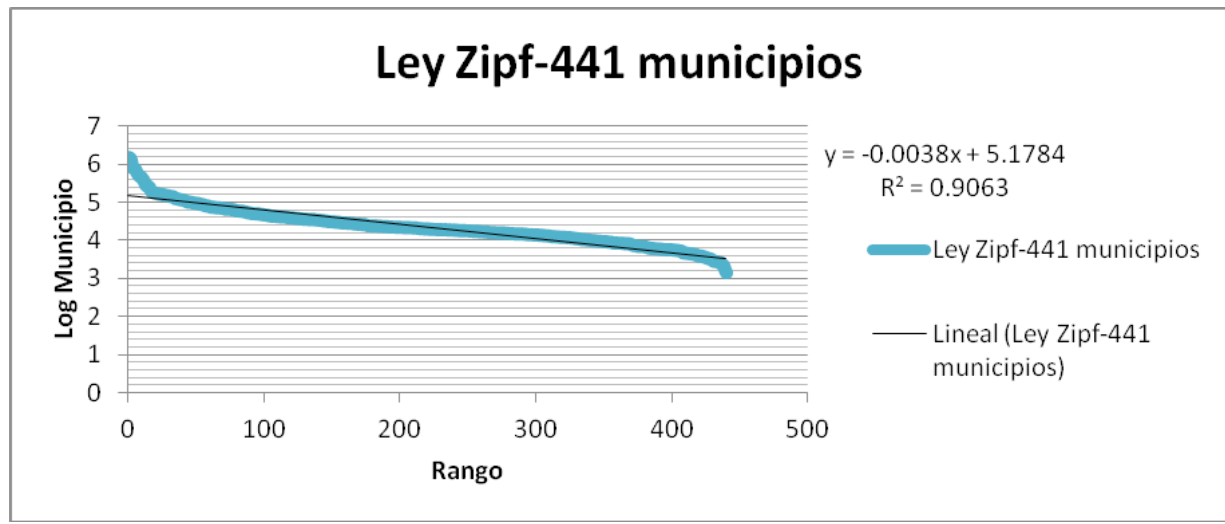

Fuente: Elaboración propia con datos de los Anuarios Estadísticos 2011 de INEGI de cada entidad federativa.

Entonces, esto nos da como resultado 41 ciudades medias, las cuales son: Acámbaro, Aguascalientes, Apatzingán, Bahía de Banderas, Celaya, Ciudad Valles, Colima, Dolores Hidalgo Cuna de la Independencia Nacional, El Salto, Fresnillo, Guadalupe, Guanajuato, Hidalgo, Irapuato, Lagos de Moreno, Lázaro Cárdenas, Manzanillo, Morelia, Pénjamo, Puerto Vallarta, Salamanca, San Felipe, San Francisco del Rincón, San Luis de la Paz, San Luis Potosí, San Miguel de Allende, Silao, Soledad de Graciano Sánchez, Tecomán, Tepatitlán de 
Morelos, Tepic, Tlajomulco de Zúńiga, Tlaquepaque, Tonalá, Uruapan, Valle de Santiago, Villa de Álvarez, Zacatecas, Zamora, Zapotlán el Grande y Zitácuaro.

Sin embargo, dentro de las 41 ciudades medias, se encuentran 3 municipios que conforman la Zona Metropolitana de Guadalajara (ZMG), los cuales son: Tlajomulco de Zúníga, Tlaquepaque y Tonalá. Por lo que dichos municipios no serán considerados como ciudades medias, con la excepción de Tonalá, puesto que aunque forma parte de la ZMG, su desarrollo urbano es menor que el de los otros municipios que la integran. En pocas palabras, Tonalá es parte de la ZMG por su cercanía a la zona conurbada, mas no por su desarrollo urbano. Entonces, Tlajomulco de Zúńiga y Tlaquepaque serán descartados como ciudades medias por ser parte de la ZMG, dado que la ZMG es considerada una ciudad grande.

Por lo tanto, dentro de la región del centro-occidente de México conformado por las 8 entidades federativas, son 39 los municipios que son considerados como ciudades medias y los cuales serán estudiados en el siguiente apartado en cuanto a su inmersión en la economía del conocimiento en la primera década del siglo XXI, con datos de las ediciones 2001 y 2011 de los Anuarios Estadísticos y la edición 2011 del Panorama Sociodemográfico de cada entidad federativa que proporciona INEGI.

Partiendo de las catalogadas como ciudades medias para fines de esta investigación, así como los principales indicadores de la economía del conocimiento de los organismos mundiales, además de los indicadores propuestos por algunos autores se tomaron los datos con base en el CENSO 2000 y CENSO 2010 para la elaboración de esta investigación, cuyos datos fueron recopilados de la página oficial de internet de INEGI y de los Anuarios Estadísticos para cada una de las entidades federativas de los ańos 2001 y 2011. Entonces se presentan en la Tabla 2 los indicadores utilizados y el motivo para utilizar cada indicador.

Tabla 2. Indicadores para la economía del conocimiento con base en la información presentada por INEGI

\begin{tabular}{|c|c|}
\hline $\begin{array}{c}\text { Indicadores Censo de } \\
\text { Población y Vivienda de } \\
\text { INEGI }\end{array}$ & Motivo por el que se utiliza el Indicador \\
\hline \multicolumn{2}{|c|}{ MEDIO AMBIENTE } \\
\hline $\begin{array}{c}\text { Superficie de áreas urbanas } \\
\text { (Kilómetros cuadrados) }\end{array}$ & $\begin{array}{c}\text { Como el mismo nombre del indicador lo dice, para analizar la } \\
\text { superficie de áreas urbanas con las que cuenta la ciudad. }\end{array}$ \\
\hline \multicolumn{2}{|c|}{ POBLACION, HOGARES Y VIVIENDA } \\
\hline \multicolumn{2}{|c|}{ Población } \\
\hline
\end{tabular}




\begin{tabular}{|c|c|}
\hline Población total & $\begin{array}{c}\text { La demografía es un punto clave en el desarrollo de la ciudad, } \\
\text { dado que es el capital humano, un activo importante dentro } \\
\text { de cualquier economía. Así mismo es el determinante para el } \\
\text { grado de urbanización de acuerdo a INEGI [2012a]. }\end{array}$ \\
\hline Población 15 ańos y más & $\begin{array}{l}\text { De acuerdo a INEGI [2012a] lo Población económicamente } \\
\text { activa comienza a los } 12 \text { ańos, pero para fines de la } \\
\text { investigación se tomará a partir de los } 15 \text { ańos y más. }\end{array}$ \\
\hline \multicolumn{2}{|r|}{ Vivienda y Urbanización } \\
\hline $\begin{array}{l}\text { Total de viviendas } \\
\text { particulares habitadas }\end{array}$ & $\begin{array}{l}\text { Indicar la cantidad de viviendas particulares habitadas que } \\
\text { existen, ya que son parte de la urbanización de la ciudad. }\end{array}$ \\
\hline $\begin{array}{l}\text { Viviendas particulares } \\
\text { habitadas que disponen de } \\
\text { energía eléctrica }\end{array}$ & \multirow{4}{*}{$\begin{array}{l}\text { Este grupo de indicadores proporcionan información } \\
\text { tanto del desarrollo y comportamiento de la urbanización } \\
\text { como de la economía del conocimiento. Los indicadores de } \\
\text { Viviendas particulares habitadas que disponen de energía } \\
\text { eléctrica, Viviendas particulares habitadas que disponen de } \\
\text { televisión, Viviendas particulares habitadas que disponen } \\
\text { de computadora y Viviendas particulares habitadas que } \\
\text { disponen de internet proporcionan información sobre la } \\
\text { economía del conocimiento. } \\
\text { De igual forma estos indicadores son parte de las TIC, por } \\
\text { lo que es por ello que son considerados como una manera de } \\
\text { indicar la presencia de la economía del conocimiento. } \\
\text { Cabe mencionar que para el indicador de Viviendas } \\
\text { particulares habitadas que disponen de internet no hay } \\
\text { datos anteriores al CENSO 2010, es decir, fue hasta dicho } \\
\text { censo que se empezó a contemplar el indicador, por lo que } \\
\text { se hará mención del dato mas no hay manera de comparar } \\
\text { su comportamiento puesto que acaba de ser incorporado. } \\
\text { Así mismo esto denota la importancia que adquirió dicho } \\
\text { instrumento de las TIC en tan poco tiempo. }\end{array}$} \\
\hline $\begin{array}{l}\text { Viviendas particulares } \\
\text { habitadas que disponen de } \\
\text { televisión }\end{array}$ & \\
\hline $\begin{array}{l}\text { Viviendas particulares } \\
\text { habitadas que disponen de } \\
\text { computadora }\end{array}$ & \\
\hline $\begin{array}{l}\text { Viviendas particulares } \\
\text { habitadas que disponen de } \\
\text { internet }\end{array}$ & \\
\hline \multicolumn{2}{|r|}{ SOCIEDAD Y GOBIERNO } \\
\hline \multicolumn{2}{|r|}{ Educación } \\
\hline $\begin{array}{l}\text { Población de } 18 \text { ańos y más } \\
\text { con nivel profesional }\end{array}$ & \multirow{4}{*}{$\begin{array}{l}\text { Estos indicadores proporcionan la especialización y la } \\
\text { calidad del recurso humano calificado para la economía del } \\
\text { conocimiento. }\end{array}$} \\
\hline $\begin{array}{l}\text { Población de } 18 \text { ańos y más } \\
\text { con posgrado }\end{array}$ & \\
\hline $\begin{array}{c}\text { Grado promedio de } \\
\text { escolaridad de la población de } \\
15 \text { y más ańos }\end{array}$ & \\
\hline $\begin{array}{l}\text { Población de } 15 \text { y más ańos } \\
\text { alfabetas }\end{array}$ & \\
\hline
\end{tabular}




\begin{tabular}{|c|c|}
\hline $\begin{array}{c}\text { Alumnos egresados en } \\
\text { secundaria }\end{array}$ & \multirow{4}{*}{$\begin{array}{l}\text { Estos indicadores proporcionan información acerca del } \\
\text { recurso humano calificado en cuanto a los alumnos egresados } \\
\text { de los ciclos escolares de 99/00 y 09/10, lo cual promueve la } \\
\text { economía del conocimiento. Además cabe mencionar que se } \\
\text { eligieron dichos niveles de educación puesto que es cuando } \\
\text { se encuentran en la edad de la Población Económicamente } \\
\text { Activa de acuerdo a INEGI [2012a]. }\end{array}$} \\
\hline $\begin{array}{l}\text { Alumnos egresados en } \\
\text { profesional técnico }\end{array}$ & \\
\hline $\begin{array}{c}\text { Alumnos egresados en } \\
\text { bachillerato }\end{array}$ & \\
\hline $\begin{array}{l}\text { Alumnos egresados en } \\
\text { formación para el trabajo }\end{array}$ & \\
\hline $\begin{array}{c}\text { Personal docente en } \\
\text { secundaria }\end{array}$ & \multirow{4}{*}{$\begin{array}{l}\text { Estos indicadores proporcionan información acerca del } \\
\text { recurso humano calificado para promover la economía del } \\
\text { conocimiento, durante los ciclos escolares de 99/00 y 09/10. } \\
\text { Además cabe mencionar que se eligieron dichos niveles de } \\
\text { educación puesto que es cuando se encuentran la edad de } \\
\text { la Población Económicamente Activa de acuerdo a INEGI } \\
\text { [2012a]. }\end{array}$} \\
\hline $\begin{array}{l}\text { Personal docente en } \\
\text { profesional técnico }\end{array}$ & \\
\hline $\begin{array}{c}\text { Personal docente en } \\
\text { bachillerato }\end{array}$ & \\
\hline $\begin{array}{l}\text { Personal docente en } \\
\text { formación para el trabajo }\end{array}$ & \\
\hline Escuelas en secundaria & \multirow{4}{*}{$\begin{array}{l}\text { Estos indicadores proporcionan información acerca de la } \\
\text { infraestructura con la que cuenta la ciudad para promover la } \\
\text { economía del conocimiento. Así mismo, dicha información es } \\
\text { de los ciclos escolares } 99 / 00 \text { y 09/10. Además cabe mencionar } \\
\text { que se eligieron dichos niveles de educación puesto } \\
\text { que es cuando se encuentran en la edad de la Población } \\
\text { Económicamente Activa de acuerdo a INEGI [2012a]. }\end{array}$} \\
\hline $\begin{array}{l}\text { Escuelas en profesional } \\
\text { técnico }\end{array}$ & \\
\hline Escuelas en bachillerato & \\
\hline $\begin{array}{c}\text { Escuelas en formación para } \\
\text { el trabajo }\end{array}$ & \\
\hline
\end{tabular}

Fuente: Elaboración propia con datos de los Anuarios Estadísticos y los Panoramas Sociodemográficos de cada entidad federativa de INEGI.

Con base en los datos recopilados y después del análisis realizado, se obtiene que la mayor parte de las ciudades medias se encuentran inmersas dentro de la economía del conocimiento. Sin embargo se encontró que hay 13 ciudades medias que no lo están, las cuales son:

1. Salamanca, Guanajuato.

2. Tepatitlán de Morelos, Jalisco.

3. Tonalá, Jalisco.

4. Zapotlán el Grande, Jalisco.

5. Hidalgo, Michoacán de Ocampo.

6. Lázaro Cárdenas, Michoacán de Ocampo.

7. Morelia, Michoacán de Ocampo.

8. Uruapan, Michoacán de Ocampo. 
9. Zamora, Michoacán de Ocampo.

10.Zitácuaro, Michoacán de Ocampo.

11.Tepic, Nayarit.

12. Ciudad Valles, San Luis Potosí.

13.Zacatecas, Zacatecas.

\section{Conclusiones}

En conclusión se encontró que de las 39 ciudades medias de la región del centro-occidente de México comprendida por 8 entidades federativas, las cuales son: Aguascalientes, Colima, Guanajuato, Jalisco, Michoacán de Ocampo, Nayarit, San Luis Potosí y Zacatecas; la mayoría se encuentran inmersas dentro de la economía del conocimiento, solamente una tercera parte no lo está.

Por lo tanto, dichas ciudades medias que no se encuentran dentro de la economía del conocimiento, se recomienda el manual de Peluffo et al. [2002]. Así mismo, es importante tomar en cuenta que no sólo es labor de las ciudades medias, sino de todo el país en conjunto, por lo que se coincide con lo encontrado por Alemán [2009, p. 49], donde menciona que:

Analizados estos casos de estudio a la luz de propuestas de desarrollo local, la territorialización de políticas nacionales en las entidades federativas puede, efectivamente, coadyuvar para pensar en nuevos escenarios de desarrollo siempre $y$ cuando se mantenga una coordinación multinivel y se vayan incorporando un número cada vez más amplio de actores en el análisis de las decisiones. Estos procesos requieren de la cooperación, asistencia técnica, recursos y control a lo largo del tiempo de todos los niveles de gobierno (nacional, estatal y supranacional).

Es así que el desarrollo local de las ciudades medias debe estar alineado al desarrollo de todo el país. Por lo tanto las estrategias locales deben ir a la par con las estrategias estatales y éstas a su vez con las estrategias nacionales, con la finalidad de promover el desarrollo uniforme. Por ello es que se considera importante la inmersión hacia la economía del conocimiento.

Por último, se concluye que la economía del conocimiento si bien es una teoría de crecimiento económico, también es una herramienta para el desarrollo urbano de la ciudad, partiendo de los estudios revisados y de la atracción de la población con conocimientos especializados hacia donde se puedan desarrollar profesionalmente, es decir, la teoría de los cluster's.

Por lo tanto, es importante considerar que la inversión hacia la economía del conocimiento se reflejará al largo plazo, dado que la educación pública comienza desde preescolar, pero rendirá frutos hasta la edad adulta, o cuando se convierte en lo catalogado como población económicamente activa. 


\section{Referencias}

Ahrweiler, P., Pyka, A., \& Gilbert, N. (2011). A New Model for University-Industry Links in Knowledge-Based Economies. Journal Of Product Innovation Management, 28(2), 218-235. Doi:10.1111/j.1540-5885.2010.00793.x

Alemán, P. (2009). Los cluster tecnológicos en México y Argentina: una estrategia para el desarrollo local. Territorios: Revista De Estudios Regionales Y Urbanos, (20/21), 3151.

Balbo, Marcello (1998). La planificación y gestión urbana local. En Jordan, Ricardo \& Simioni, Daniela (comp.). Ciudades intermedias de América Latina y el Caribe: Propuestas para la gestión urbana (pp. 107-126). Santiago de Chile: Naciones Unidas.

Banco Mundial (2000). Ciudades en transición. Resumen. Estrategia del Banco Mundial ara los gobiernos urbanos y locales. Washington, D.C.: Banco Internacional de Reconstrucción y Fomento/Banco Mundial.

Barković, I., Borozan, Đ., \& Dabić, M. (2008). Emerging Opportunities and Challenges for Universities in the Knowledge-Based Economy: the Croatian Perspective. Proceedings Of The Northeast Business \& Economics Association, 29-36.

Cabrales, Luis Felipe \& Canosa, Elia (2001). América Latina, ciudades cerradas. Revista de hechos e ideas, 83, 95-109, Madrid.

Carrillo Gamboa (2011). Generación de valor con base en conocimiento, en Martínez Sánchez, América \& Corrales Estrada, Martha (2011). Administración del conocimiento $y$ desarrollo basado en conocimiento. México: Cengage Learning Editores.

Castells, Manuel (1999). Globalización, Identidad y Estado en América Latina. Santiago de Chile: Programa de las Naciones Unidas para el Desarrollo.

Celis, A. (2009). El top ten de las ciudades, una crítica de la medición de la competitividad municipal y urbana. (Spanish). Problemas Del Desarrollo. Revista Latinoamericana De Economía, 40(159), 169-190.

Chivu, I., Popescu, D., Scarlat, C., Ciocarlan-Chitucea, A., \& Popescu, D. (2010). Human resources development within romanian small and medium sized enterprises in a knowledge-based economy. Metalurgia International, 15(7), 48-52.

Cuervo González, Luis Mauricio (2003). Ciudad y globalización en América Latina: estado del arte. Santiago de Chile: Naciones Unidas.

Cuervo González, Luis Mauricio (2004). Desarrollo económico y primacía urbana en América Latina: Una visión histórico-comparativa, en Torres Ribeiro, Ana Clara (comp.) (2004). El rostro urbano de América Latina. O rosto urbano da América Latina. Buenos Aires: CLACSO.

Cuervo, González, Luis Mauricio (2006). Globalización y territorio. Santiago de Chile: Naciones Unidas.

Ding, C., \& Zhao, X. (2011). Assessment of Urban Spatial-Growth Patterns in China during Rapid Urbanization. Chinese Economy, 44(1), 46-71.

Ferraro, Ricardo. (1999). La marcha de los locos: Entre las nuevas tareas, los nuevos empleos 
y las nuevas empresas. Argentina: Fondo de Cultura Económica.

INEGI (2001). Anuarios Estadísticos correspondientes a cada entidad federativa: Aguascalientes, Colima, Guanajuato, Jalisco, Michoacán de Ocampo, Nayarit, San Luis Potosí y Zacatecas.

INEGI (2011a). Anuarios Estadísticos correspondientes a cada entidad federativa: Aguascalientes, Colima, Guanajuato, Jalisco, Michoacán de Ocampo, Nayarit, San Luis Potosí y Zacatecas.

INEGI (2011b). Panoramas Sociodemográfico correspondientes a cada entidad federativa: Aguascalientes, Colima, Guanajuato, Jalisco, Michoacán de Ocampo, Nayarit, San Luis Potosí y Zacatecas. México: INEGI.

INEGI (2012). Glosario de INEGI. Recuperado de: http://www.inegi.org.mx/ est/contenidos/espanol/proyectos/encuestas/hogares/ene/metadatos/PEA. asp?s=est\&c=10690. Revisado el 5 de septiembre de 2012.

Jordan, Ricardo \& Simioni, Daniela (compiladores) (1998). Ciudades intermedias de América Latina y el Caribe: Propuestas para la gestión urbana. Santiago de Chile: Naciones Unidas.

Kriđčiünas, K., \& Daugëlienë, R. (2006). The Assessment Models of Knowledge-Based Economy Penetration. Engineering Economics, 50(5), 36-46

Kuznetsov, Yevgeny \& Dahlman, Carl. J. (2008). Mexico's Transition to a KnowledgeBased Economy: Challenges and Opportunities. Washington, DC.

Macció, Guillermo A. (1985). Diccionario demográfico multilingüe. Versión en espańol. Bélgica: Ordina Editions \& CELADE.

Medina Vásquez, Javier \& Ortegón, Edgar (2006). Manual de prospectiva y decisión estratégica: bases teóricas e instrumentos para América Latina y el Caribe. Santiago de Chile: Naciones Unidas.

Melnikas, B. (2009). Sustainable development and creation of the knowledge based society: the new challenges and strategic decisions. Public Administration (16484541), 2(22), 6-14.

O’Hagan, S., \& Rutland, T. (2008). A Comparison of Canada's Small, Medium, and Large Cities in the Knowledge Economy. Canadian Journal Of Urban Research, 17(1), 130-154.

Organización para la Cooperación y el Desarrollo Económico (1996). The knowledgebased economy. Francia: OCDE.

Peluffo, Martha Beatriz \& Catalán Contreras, Edith (2002). Introducción a la gestión del conocimiento y su aplicación al sector público. Santiago de Chile: Naciones Unidas.

Romeiro, Patricia \& Méndez Gutiérrez Del Valle, Ricardo (2008). Las ciudades del conocimiento: revisión crítica y posibilidades de aplicación de las ciudades intermedias. Script Noca. Revista Electrónica de Geografía y Ciencias Sociales. 12(270). ISSN: 1138-9788

Rybakovas, E. (2010). The Alternative Resources of the Knowledge-Based Economy 
Development. Social Sciences (1392-0758), 67(1), 67-79

Sabatini, Francisco (1998). Direcciones para el futuro. En Jordan, Ricardo \& Simioni, Daniela (comp.). Ciudades intermedias de América Latina y el Caribe: Propuestas para la gestión urbana (pp. 127-214). Santiago de Chile: Naciones Unidas.

Sakaiya, Taichi (1995). Historia del futuro: La sociedad del conocimiento. Chile: Editorial Andrés Bello.

Schneier, G. (1990). Latin America: a tale of cities. International Social Science Journal, 42(3), 337.

Sîrbu, M., Doinea, O., \& Mangra, M. (2009). Knowledge based economy- the basis for insuring a sustainable development. Annals Of The University Of Petrosani Economics, 9(4), 227-232

Soria, Manuel \& Aboites, Jaime. (2008). Economía del conocimiento y propiedad intelectual: Lecciones para la economía mexicana. México: Siglo XXI Editores.

Toma, Viorica (2010). European Union strategy for building a knowledge-based economy. Metalurgia International, 15134-138

Welfe, W. (2008). A Knowledge-Based Economy: New Directions of Macromodelling. International Advances In Economic Research, 14(2), 167-180. Doi:10.1007/s11294008-9143-1

Zientara, P. (2008). Polish regions in the age of a knowledge-based economy. International journal of urban \& regional research, 32(1), 60-85. Doi:10.1111/j.14682427.2008.00776.x

Zoido, Florencio; De la Vega, Sofía; Morales, Guillermo; Mas, Rafael \& Lois, Rubén. (2000). Diccionario de geografía urbana, urbanismo y ordenación del territorio. Espańa: Editorial Ariel S.A. 